\title{
The six geometries revisited
}

\author{
Austin Kang ${ }^{\mathrm{a}}$ (10) \\ Marino Musiliti \\ Mauro Farella ${ }^{\mathrm{a}, \mathrm{c}}$
}

${ }^{a}$ Discipline of Orthodontics, Department of Oral Sciences, Faculty of Dentistry, University of Otago, Dunedin, New Zealand

${ }^{\text {b} P r i v a t e ~ P r a c t i c e, ~ S a l e r n o, ~ I t a l y ~}$

'Sir John Walsh Research Institute, Faculty of Dentistry, University of Otago, Dunedin, New Zealand
Forces and moments delivered by a straight wire connecting two orthodontic brackets are statically indeterminate and cannot be estimated using the classical equations of static equilibrium. To identify the mechanics of such two-bracket systems, Burstone and Koenig used the principles of linear beam theory to estimate the resulting force systems. In the original publication, however, it remains unclear how the force systems were calculated because no reference or computational details on the underlying principles have been provided. Using the moment carry-over principle and the relative angulation of the brackets, a formula was derived to calculate the relative moments of the two brackets. Because of the moment equilibrium, the vertical forces that exist as a forcecouple on the two brackets can also be calculated. The accuracy of the proposed approach can be validated using previously published empirical data.

[Korean J Orthod 2020;50(5):356-359]

Key words: Tooth movement, Orthodontics, Biomechanics, Bracket geometries
Received July 31, 2019; Revised October 31, 2019; Accepted November 19, 2019.

Corresponding author: Austin Kang.

Professional Practice Fellow, Discipline of Orthodontics, Department of Oral Sciences, Faculty of Dentistry, University of Otago, Walsh Building, 310 Great King Street, PO Box 56, Dunedin 9054, New Zealand.

Tel +64-3-479-7068 e-mail kaaang@mensa.org.nz

How to cite this article: Kang A, Musilli M, Farella M. The six geometries revisited. Korean J Orthod 2020;50:356-359.

(C) 2020 The Korean Association of Orthodontists.

This is an Open Access article distributed under the terms of the Creative Commons Attribution Non-Commercial License (http://creativecommons.org/licenses/by-nc/4.0) which permits unrestricted non-commercial use, distribution, and reproduction in any medium, provided the original work is properly cited. 


\section{INTRODUCTION}

A straight wire connecting two orthodontic brackets produces a statically indeterminate force system. Therefore, the nature and magnitude of the forces and moments delivered at each bracket are unknown and cannot be calculated using the classical equations of static equilibrium.'

To identify the working mechanics of such twobracket systems, the principles of linear beam theory were used to calculate the resulting force systems using a computer algorithm. ${ }^{2}$ The absolute magnitude of the force systems generated on each bracket depended on the properties of the wire, distance between the brackets, and bracket angulations. However, the bracket angulations alone determined the relative magnitude of the force systems between the two brackets. Six different bracket angulations were identified and the relative forces and moments on each of the two brackets had been summarized as the six geometries. ${ }^{2}$ By assessing the ratio between the bracket angles in a given clinical situation, it is possible to match the configuration of the brackets with one of the six identified geometries, and thereby predict the ratios of the delivered moments and forces between the two brackets. The original publication on the six geometries by Burstone and Koenig ${ }^{2}$ has neither provided the computational details of the relative forces and moments nor provided a reference for the underlying principles. A formula to calculate the six geometries as a continuum was recently described in a book chapter by Burstone and Choy; ${ }^{3}$ however, there were no details on the theoretical framework used for computation.

An elastic wire connecting two brackets is an example of a statically indeterminate beam, whose relative force systems can be analyzed with different computational methods. One such method is the mechanism of moment distribution, ${ }^{4}$ which involves consideration of the carried-over moments.

This letter aims to revisit the landmark article from Burstone and Koenig ${ }^{2}$ taking the carried-over moments into consideration. Using this theoretical framework, a simple formula can be derived and validated using the data provided in the original publication. This formula can be used to calculate the relative force systems for any given bracket angulation.

\section{MOMENTS}

In a two-bracket system, the moments at each bracket are proportional to the bracket angulation (Figure 1A).

\section{MOMENT CARRY-OVER}

Moment carry-over is a phenomenon exhibited by an elastic beam (or wire) that occurs when multiple moments coexist. The neighboring moments influence each other; in classical mechanics, this fact has been used to calculate statically indeterminate systems. A proportion of each moment gets carried over to the neighboring moment depending on the flexural stiffness of an elastic beam and the number of moments existing in the vicinity.

In a two-bracket system, the moment of each bracket gets carried over to the adjacent bracket in the same direction and with half the magnitude (Figure $1 \mathrm{~B}$ and $1 \mathrm{C}$ ).

Thus, the moment delivered to a bracket depends on the bracket's angulation and half the angulation of the neighboring bracket:

$$
\begin{aligned}
& M_{A} \alpha \theta_{A}+\frac{1}{2} \theta_{B} \\
& M_{B} \alpha \theta_{B}+\frac{1}{2} \theta_{A}
\end{aligned}
$$

Therefore, the following formula was derived for calculating the relative moments of the two brackets:
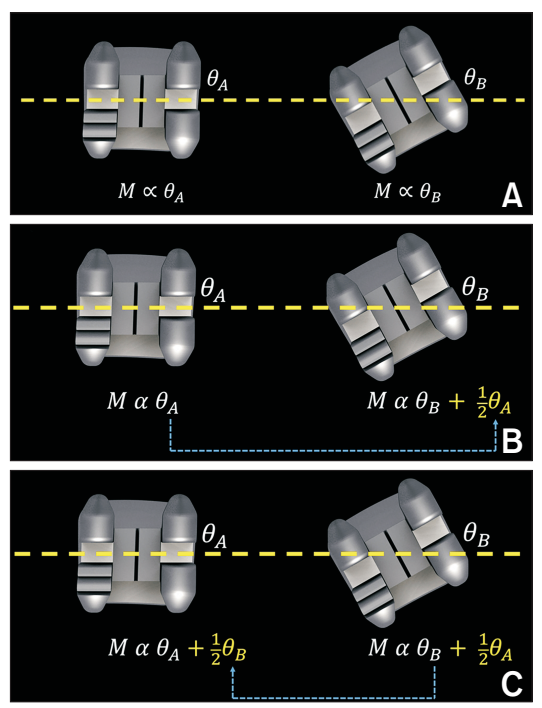

Figure 1. A, Moments delivered in a two-bracket system are proportional to the angulation of each bracket. $\mathbf{B}$, The carry-over effect of bracket $A$ affecting bracket $B$ in the same direction and with half the magnitude. $C$, The carryover effect of bracket $B$ affecting bracket $A$.

$M$, Moment; $\theta_{A}$ angulation of bracket $A ; \theta_{B}$, angulation of bracket B. 


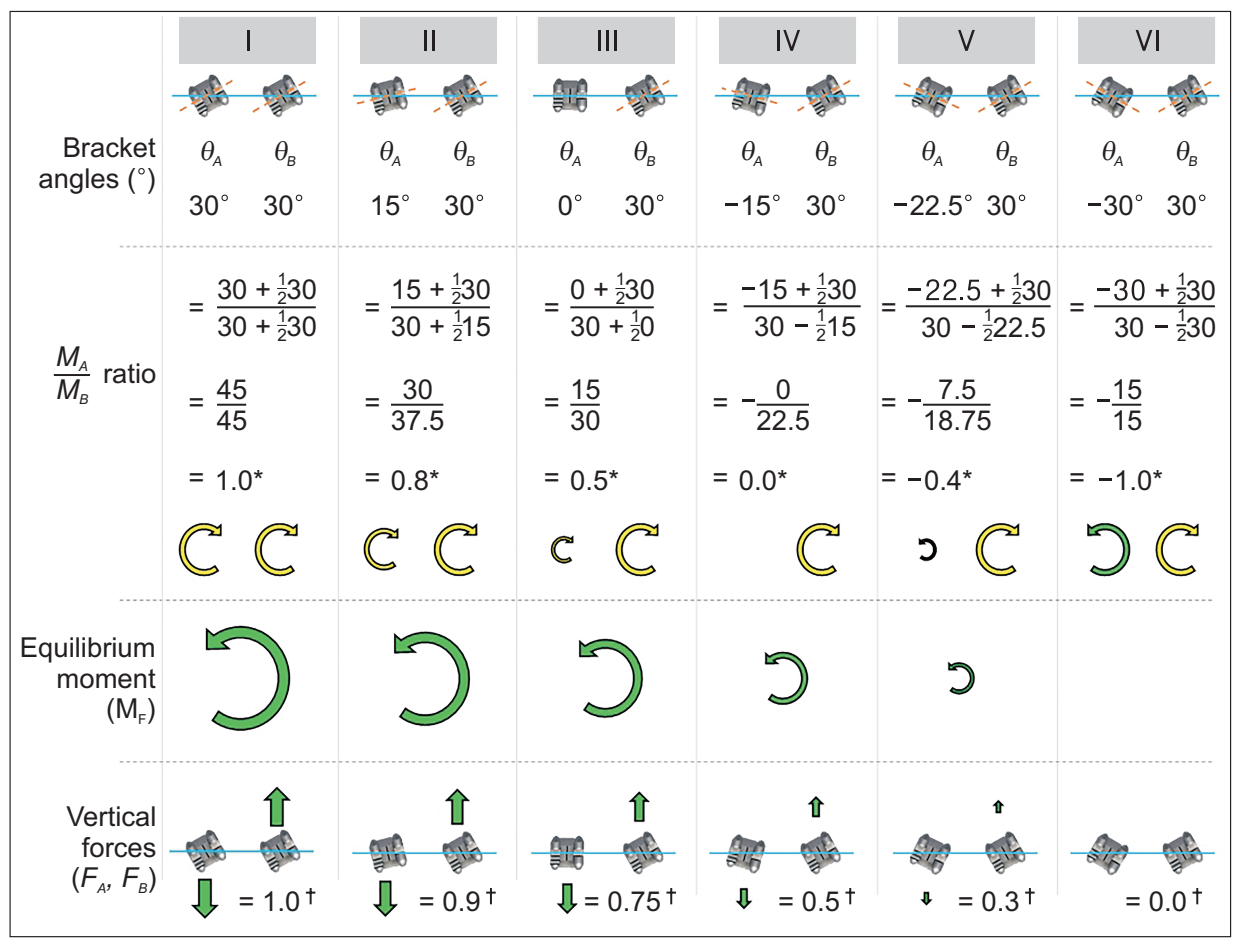

Figure 2. Computational approach to estimate the moment ratios $\frac{M_{A}}{M_{B}}$ for different two-bracket geometries as described by Burstone and Koenig. ${ }^{2}$

A, Left bracket; $B$, right bracket; $\theta_{A}$ angulation of bracket $A_{i} \theta_{B}$, angulation of bracket $B$. *Note that the estimated ratios for the six geometries are identical to those reported in the original article.

${ }^{\dagger}$ Force magnitudes are relative to the those produced in Geometry I when all other conditions are kept constant.

$$
\frac{M_{A}}{M_{B}}=\frac{\theta_{A}+\frac{1}{2} \theta_{B}}{\theta_{B}+\frac{1}{2} \theta_{A}}
$$

Using the formula above, the moments of the twobracket systems can be calculated with the known angulation of the two brackets $\left(\theta_{A}\right.$ and $\left.\theta_{B}\right)$, and the formula is comparable to that described by Burstone and Choy. ${ }^{3}$ Figure 2 shows examples of the six geometries and the method by which the formula can be used to calculate the relative moments on the brackets in each case.

\section{VERTICAL FORCES (MOMENT-EQUILIBRIUM)}

As discussed in the original article, the moments of the six geometries are at equilibrium with equal magnitudes of the clockwise and counter-clockwise moments (Figure 3A and 3B).

Only the moments of the two brackets in Geometry $\mathrm{V} l$ exist in an equilibrium:

$$
M_{A}+M_{B}=0
$$

For Geometries $1-\mathrm{V}$, an external moment is required for the system to be in equilibrium:

$$
M_{A}+M_{B}+M_{F}=0
$$

This moment, $\mathrm{M}_{\mathrm{F}}$, results from the vertical forces on the two brackets, which exists as a force-couple (Figure
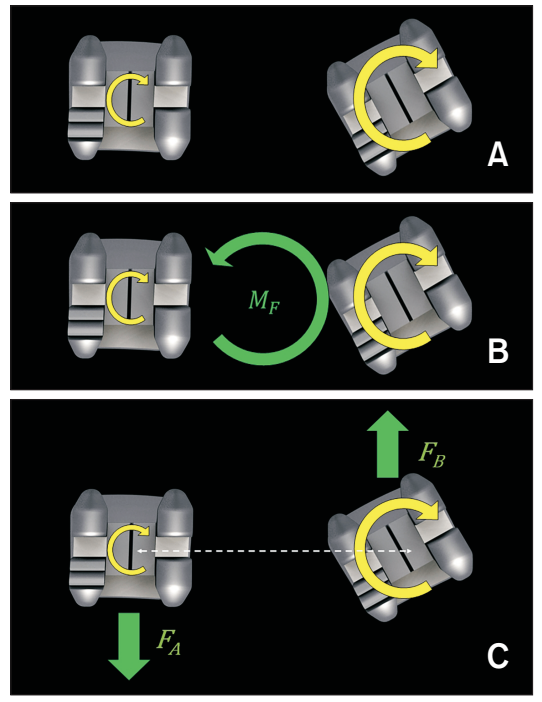

Figure 3. A, Moments delivered to each bracket. B, Equilibrium moment existing in the counter-clockwise direction to achieve moment equilibrium. C, Equilibrium moment existing as force couple to produce vertical forces on the brackets.

$M_{F r}$ Equilibrium moment; $F_{A}$, vertical force acting on bracket $A_{i} F_{B}$ vertical force on bracket $B$.

3C). Figure 2 shows how the relationship above can be used to estimate the relative forces acting on the two brackets for each of the six geometries. 


\section{FORCE SYSTEMS PRODUCED BY A STRAIGHT WIRE ON THREE OR MORE BRACKETS}

For three or more brackets, the moment on each bracket is carried over to all the other neighboring brackets in the system. However, the magnitude and direction of these carried-over moments differ for each bracket, similar to the behavior of the carried-over moments in statically indeterminate systems derived with the moment distribution method.

The scope of this report is limited to two-bracket systems as three or more bracket systems involve additional complex theoretical and mathematical concepts.

\section{CONCLUSION}

Using carry-over moments, a simple formula can be derived for calculating the moment and force ratios produced by a straight wire in a two-bracket system for any bracket angulation, including the transitional stages between the various geometries. This formula can be used to facilitate the appraisal of the six geometries for educational and clinical purposes.

\section{CONFLICTS OF INTEREST}

No potential conflict of interest relevant to this article was reported.

\section{REFERENCES}

1. Lindauer J. The basics of orthodontic mechanics. Semin Orthod 2001;7:2-15.

2. Burstone $\mathrm{CJ}$, Koenig HA. Force systems from an ideal arch. Am J Orthod 1974;65:270-89.

3. Burstone CJ, Choy K. Forces from wires and brackets. In: Burstone CJ, Choy K, eds. The biomechanical foundation of clinical orthodontics. Chicago: Quintessence Publishing; 2015. p. 332.

4. Cross H. Analysis of continuous frames by distributing fixed-end moments. Paper presented at: American Society of Civil Engineers Annual Convention; 1930 Jul 9-11; Cleveland, USA. New York: American Society of Civil Engineers, 1930. p. 919-28. 